\title{
The diagnostic capability of electrocardiography on the cardiogenic shock in the patients with acute myocarditis
}

\author{
Dan Yang ${ }^{1 \dagger}$, Qing Dai ${ }^{1 \dagger}$, Han Wu $^{1 \dagger}$, Jianzhou Chen ${ }^{1}$, Jingmei Zhang ${ }^{1,2^{*}}$ and Zhonghai Wei ${ }^{1 *}$ (D)
}

\begin{abstract}
Background: The study was performed to assess the diagnostic capability of ECG on the cardiogenic shock (CS) in acute myocarditis. A new score was derived from the combination of the ECG parameters and the diagnostic value was also evaluated.
\end{abstract}

Methods: Total 103 consecutive patients with acute myocarditis admitted in Nanjing Drum Hospital were enrolled in the current study. The cohort was divided into fulminant myocarditis group ( $F M, n=20)$ and non fulminant myocarditis group ( $N F M, n=83$ ). The demographic features, results of electrocardiography (ECG) and ultracardiography were compared. Logistic regression analysis was conducted to identify the relevant factors in ECG parameters. We created a new variable called "ECG score" by certain combination of ECG parameters. The diagnostic capability of ECG score for CS was compared with the existing diagnostic indices using regression model and receiver-operating characteristics (ROC) analysis.

Results: There were several changes on ECG significantly different between the two groups. Multivariate regression analysis demonstrated $\mathrm{PR}+\mathrm{QRS}$ interval $(P=0.008)$, ventricular arrhythmia $(P=0.001)$ and pathological $\mathrm{Q}$ wave $(P=0.003)$ were the independent relevant factors of CS. The derived variable "ECG score" was identified as a significant relevant factor of CS by multivariate regression model. ROC analysis showed PR+QRS interval, ventricular arrhythmia and pathological Q wave all had equivalent diagnostic capability to left ventricular ejection fraction (LVEF) and shock index (SI). ECG score was equivalent to LVEF but superior to SI in diagnosing CS

Conclusions: ECG was valuable in diagnosing CS due to acute myocarditis. The ECG score was superior to the traditional diagnostic indices and could be used for an rapid recognition of CS.

Keywords: Fulminant myocarditis, Cardiogenic shock, Electrocardiography, Diagnosis

\section{Background}

Myocarditis is a inflammatory disease involving injury of the cardiac myocytes, the incidence of which is estimated approximate $0.02-0.1 \%$ in the general population

\footnotetext{
*Correspondence: weizhnjjs@yeah.net; zhangjm01@163.com

†Dan Yang, Qing Dai and Han Wu have contributed equally to this manuscript

${ }^{1}$ Department of Cardiology, Drum Tower Hospital, Medical School of Nanjing University, Nanjing 210008, China

Full list of author information is available at the end of the article
}

$[1,2]$. Fulminant myocarditis (FM), the most severe type, is characterized with cardiogenic shock (CS) and haemodynamical disorder. It usually undergoes a fatal course and some of the cases probably come to a frustrating end. On the contrary, nonfulminant myocarditis (NFM) often produces symptoms of heart failure with stable haemodynamical status [3]. Of note, NFM could sometimes evolve into FM very quickly after first medical contact (FMC). It has been reported that FM patients have more likelihood of death or heart transplantation $[4,5]$. Aggressive 
treatment including inotropic agents, mechanical circulatory support would help the patients get through the CS stage and improve the prognosis [6]. Thus, quick recognition of the upcoming or existing CS is essential.

As far as we know, CS has an arbitrary definition of systolic blood pressure below $90 \mathrm{mmHg}$ with inadequate peripheral perfusion. The diagnostic accuracy is influenced by the basic level of the blood pressure. Shock index (SI) is a more widely used for prediction or diagnosis of shock. Different cutoff values have been suggested, which reveals that the diagnostic capability of SI probably varies under different clinical conditions [7-10]. Therefore, more diagnostic measures are needed for diagnosing the CS in the presence of myocarditis.

It has been observed that the different components of electrocardiography (ECG) usually alter in myocarditis patients, such as prolonged PR interval, wide QRS complex, nonspecific ST-T changes, emergence of pathological Q wave [11]. Although many changes are not pathognomonic, a few features are more likely to occur in the FM than NFM [12-15]. Based on the above findings, we postulate that combination of the various alterations on the ECG might create an useful index for rapid diagnosis of the CS in acute myocarditis patients.

\section{Methods}

\section{Study population}

All the patients who were suspected of acute myocarditis were admitted in Nanjing Drum Hospital, Medical School of Nanjing University from November 2010 to September 2019. The inclusion criteria were as follows: (1) The included paitents had no age limitation; (2) The symptom onset was less than 1 week before admission; (3) The diagnosis of acute myocarditis was confirmed after clinical, laboratory tests and imaging evaluation; (4) The whole data were available. The exclusions criteria were as follows: (1) The symptom onset was above 1 week before addmission; (2) The myocardial injury was due to other heart diseases, such as worsening of heart failure, acute coronary syndrome (ACS), arrhythmia, cardiomyopathy; (3) The myocardial injury was caused by other systemic diseases, such as tumor, immune disorder, hematological disease, stroke; (4) The patients suffered from acute pericarditis without myocardial injury; (5) The clinical data were not accessible.

Total 168 patients suspicious of acute myocarditis were put into initial analysis. Acute myocarditis was diagnosed based on the following clinical presentations and auxiliary tests [16]: (1) the history of flu-like prodromes including respiratory or gastrointestinal symptoms; (2) acute onset of symptoms such as chest discomfort, palpitation, shortness of breath, fatigue, etc.; (3) elevation of myocardial injury biomarkers; (4) various changes on
ECG including non-specific ST-T alteration, ACS pattern, pericarditis pattern, atrioventricular block (AVB), etc.; (5) structure or function abnormality on ultracardiography (UCG); (6) exclusion of coronary heart disease (CHD) or present CHD not responsible for the above manifestations. Besides, cardiac magnetic resonance (CMR) was performed for some patients after consideration of the individual conditions. CMR could provide the pathognomonic features for confirming the diagnosis and evaluation of the myocardial injury. FM could be diagnosed when the cardiac function was severely impaired which led to CS with or without fatal ventricular arrhythmia [17]. CS was defined as systolic blood pressure less than $90 \mathrm{mmHg}$ for at least $30 \mathrm{~min}$, which presented clinical signs of pulmonary congestion and peripheral hypoperfusion or needed inotropic agents to maintain the blood pressure level. After series of evalutions, 12 patients were exluded due to pericarditis without myocardial injury. 41 patients were excluded due to other heart diseases (ACS, tachyarrhythmia, heart failure, etc.). 9 patients were excluded due to other systemic disease (immune disorder, stroke, hematological diseases, etc.). 3 patients were excluded due to the symptom onset more than 1 week before admission. Finally, 103 patients were eligible for the study cohort (Fig. 1).

The study cohort had a median age of 30 years (IQR: $22-46$ years) and contained 40 female patients $(38.8 \%)$ and 63 male patients (61.2\%). There were 20 patients (19.4\%) with diagnosis of FM and the rest were NFM. The study cohort was divided into FM group and NFM group for further analysis. Data was obtained from the databases in our institution and the ethics has been approved by the Medical Ethics Committee of Nanjing Drum Tower Hospital, Medical School of Nanjing University (2019-190-01).

\section{Patient management}

All the patients were admitted into cardiac care unit (CCU) for monitoring the potential adverse cardiac events. Bedside UCG was accomplished within $24 \mathrm{~h}$ after hospitalization. If the patients were suspicious of ACS according to the risk factors, symptom, alterations on ECG, etc., angiography was performed after admission. CMR was taken if necessary for confirming the diagnosis or assessing the extent of myocardial injury. If the patients had symptoms of cardiac dysfunction but with haemodynamical stability, the diuretics, $\beta$ adrenergic blockade, angiotensin-converting enzyme inhibitor (ACEI)/angiotensin receptor blockade (ARB) and aldosterone antagonist were considered for individual therapy [18]. As to the patients with fulminant type, measurements for reversing the haemodynamical derangement were carried out as soon as possible. Intra-aortic balloon 


\section{8 patients were initially suspected of acute myocarditis

Exclusions:
- 12 patients due to acute pericarditis without myocardial injury
- 25 patients due to worsening of heart failure with infection
- 6 patients due to acute coronary syndrome
- 8 patients due to tachyarrhythmia
- 9 patients due to cardiomyopathy
hematological disease, etc)
- 3 patients due to symptom onset more than 1 week before
admission
103 patients were enrolled in the current study eventually
NFM group(n=83) vs. FM group(n=20)

Fig. 1 Flow chart describing the enrollment of the patient cohort. NFM nonfulminant myocarditis, FM fulminant myocarditis

pump (IABP) was the first option of mechanical circulatory support. The inotropic agents were considered in combination with IABP. Noninvasive respiratory ventilation was utilized for alleviating the symptoms of dyspnea due to pulmonary edema. If the haemodynamical status could not be stabilized after the support of IABP and noninvasive ventilation, extracorporeal membrane oxygenation (ECMO) and invasive ventilation were performed. Swan-Ganz catheter was deployed for haemodynamical monitor according to the individual conditions. The temporary pacemaker was inserted if there was second to third degree AVB. Prolonged PR interval combined with wide QRS complex on the ECG might suggest a high likelihood of upcoming complete heart block, prophylactic temporary pacemaker was therefore used. Ventricular tachycardia (VT) was life-threatening in fulminant myocarditis. Defibrillation and/or overdriving by temporary pacemaker were helpful for termination of sustained VT and aminodarone was administrated for control of the malignant ventricular tachycardia.

\section{Statistical analysis}

Continuous variables were shown as mean \pm standard deviation (mean $\pm S D$ ) if they were normally distributed, whereas they were presented as median and interquartile range (IQR) if they were skewed distributed. Categorical variables were shown as frequencies and percentages. The comparison of the continuous variables between the two groups was performed using T-test or Wilcoxon rank-sum test. The comparison of the categorical variables between the two groups was performed using $\chi^{2}$ test or Fisher exact test. The potential predictors of CS, selected from demographic features, ECG parameters and UCG parameters, were analyzed using univariate logistic regression initially. Considered the interest of ECG changes in the current study, we picked up the ECG parameters with $P<0.10$ for multivariate logistic regression analysis in order to identify the significant relevant factors. After the multivariate analysis, the significant ECG parameters were integrated to derive a new variable (we called ECG score). This derived ECG score together with other covariates were set in the multivariate logistic regression model for evaluation of the odd ratio. In the multivariate regression model, the variables were selected using backwards method. The regression models were calibrated with Hosmer-Lemeshow $\chi^{2}$ test for the goodness of fit. The correlation analysis was carried out using Pearson coefficient $(r)$ or Spearman coefficient $(\rho)$ for the colinearity tests of the covariates. The diagnostic capability of the significant variables was tested with receiver-operating characteristics (ROC) analysis. The area under curve (AUC) was calculated to compare the diagnostic capability of the ECG score with the traditional diagnostic indices. Specificity, sensitivity, accuracy, positive predictive value (PPV) and negative predictive value (NPV) were analyzed for identification of a reasonable cutoff value. The statistical analysis was performed by Stata version 12.0 (StataCop., College Station, Texus, USA). All the tests were 2 sided. Values of $P<0.05$ were considered statistically significant. 


\section{Results}

The demographic characteristics of the study cohort The median age of the patients in the FM group was numerically higher than that of the patients in NFM group with a trend towards statistical significance. In the FM group, female patients accounted for a much higher proportion. Meanwhile, 17 patients $(85 \%)$ had presented CS when admitted in the emergency department (ED), while 3 patients (15\%) developed CS on the second day after hospitalization. FM patients had also higher level of myocardial injury biomarkers, inflammatory biomarkers, brain natriuretic peptide (BNP) and hepatic transaminase (Table 1).

\section{ECG and UCG findings of the study cohort}

The PR intervals and QRS complex duration in the FM group were prolonged significantly than those in NFM group. There were also more patients in the FM group had pathological Q wave and ST-T alterations. Ventricular arrhythmia, including frequent non-sustained VT, sustained VT and accelerated idioventricular rhythm (AIVR) was overwhelming in the FM patients. As to $\mathrm{AVB}$, the incidence of first and third degree AVB was much higher in FM patients (Table 2). Of note, the three patients, who developed CS on the second day after hospitalization, presented nearly normal left ventricular systolic function but abnormal ECG on the first day. One patient had prolonged PR interval, pathological Q wave and non-sustained VT. Another one had prolonged PR interval and wide QRS complex. The last one had prolonged PR interval, wide QRS complex and pathological $\mathrm{Q}$ wave.

The ventricular wall thickness seemed to be higher in the FM group numerically with a trend towards statistical significance. The left ventricular end-diastolic diameter (LVEDD) was similar while the left ventricular end-systolic diameter (LVESD) was significant lower in the NFM group. The FM patients had a worse left ventricular ejection fraction (LVEF) due to the severe impaired cardiac function (Table 2).

\section{Establishment of logistic regression model}

The univariate logistic regression analysis was carried out for identification of the potential relevant factors for CS. The significant variables were listed in the Table 3. Of note, two groups of variables had colinearity, LVEF and LVESD $(\rho=-0.76, P<0.01)$, intraventricular septal thickness (IVST) and left ventricular posterior wall thickness (LVPWT) $(\rho=0.95, P<0.01)$. Thus, LVESD and LVPWT were not used for regression analysis.

We performed the multivariate logistic regression for the eligible ECG parameters elected from the univariate analysis firstly in order to identify the independent relevant factors. For simplification, PR interval and QRS complex duration were added together to become a single variable: $\mathrm{PR}+\mathrm{QRS}$ interval. This integrated variable together with pathological Q wave, ST segment elevation, ST segment depression, $\mathrm{T}$ wave inverse, ventricular arrhythmia and second to third degree AVB were set in the multivariate analysis. First degree AVB was excluded from multivariate analysis for sake of a certain overlap with PR interval. Consequently, PR+QRS interval, pathological $Q$ wave and ventricular arrhythmia were confirmed as independent relevant factors (Table 4). Hosmer-Lemeshow $X^{2}$ test suggested a excellent model fit $\left(X^{2}=1.92, P=0.59\right)$. The diagnostic capability of the three factors together with LVEF and SI were evaluated using ROC (Fig. 2). LVEF had the largest AUC whereas ventricular arrhythmia had the smallest. Nevertheless, there was no significant difference in the AUC of the five indices.

\section{Derivation of new predictor and model fit}

In order to promote the diagnostic capability of the three relevant factors elected from ECG, we conceived a new variable generated by a certain combination of the three variables. First, we denoted scores to each factor. PR interval and QRS duration were assigned the equivalent score to their actual values. For example, PR interval is given 150 points if it is $150 \mathrm{~ms}$. QRS complex duration is given 140 points if it is $140 \mathrm{~ms}$. PR + QRS interval is the sum of each score. Therefore, the score of PR+QRS interval in the above example is 290 points. Ventricular arrhythmia is denoted 1 point if it occurs while 0 point if it does not occur. Pathological Q wave is denoted the score in the same way. Second, we define the derived new variable. We call it "ECG score" for the present time and ECG score $=(P R$ interval $+Q R S$ complex duration $) \times($ ventricular arrhythmia + pathological $Q$ wave +1 ). For another example, if PR interval $=200 \mathrm{~ms}$, QRS complex duration $=120 \mathrm{~ms}$, ventricular tachycardia occurs without pathological Q waves existence, ECG sco re $=(200+120) \times(1+0+1)=640$ points. We provide some ECG of acute myocarditis in Fig. 3 to illustrate this method.

Now the ECG score should be drawn in the multivariate regression model for assessing the odd ratio. The results of the model fit were listed in the Table 5. In the Model 1, ECG score and LVEF were identified as the significant relevant factors of CS $\left(\chi^{2}=0.48\right.$, $P=0.92$ for Hosmer-Lemeshow test). Model 2 was quite similar to the Model 1 but without LVEF. Consequently, ECG score and SI were the significant relevant factors in the Model $2\left(x^{2}=1.68, P=0.64\right.$ for HosmerLemeshow test). The ROC of ECG score, LVEF and SI 
Table 1 The baseline characteristics of patient cohort

\begin{tabular}{|c|c|c|c|}
\hline & FM group $(n=20)$ & NFM group $(n=83)$ & $P$ value \\
\hline Age (year) & $36(29-48)$ & $28(22-44)$ & 0.06 \\
\hline Female & $12(60 \%)$ & $28(33.7 \%)$ & 0.03 \\
\hline Hypertension & $1(5 \%)$ & $8(9.6 \%)$ & 1.00 \\
\hline Diabetes & 0 & $3(3.6 \%)$ & 1.00 \\
\hline Coronary heart disease & 0 & $1(1.2 \%)$ & 1.00 \\
\hline Smoker & $4(20 \%)$ & $9(10.8 \%)$ & 0.27 \\
\hline Drinker & $1(5 \%)$ & $6(7.2 \%)$ & 1.00 \\
\hline $\mathrm{BMI}\left(\mathrm{kg} / \mathrm{m}^{2}\right)$ & $24.4 \pm 2.87$ & $23.0 \pm 3.19$ & 0.15 \\
\hline \multicolumn{4}{|l|}{ Onset symptom } \\
\hline Chest discomfort & $15(85 \%)$ & $64(77.1 \%)$ & 0.55 \\
\hline Fatigue & $6(30 \%)$ & $12(14.5 \%)$ & 0.11 \\
\hline Palpitation & $2(10 \%)$ & $14(16.9 \%)$ & 0.73 \\
\hline Cough & $5(25 \%)$ & $11(13.3 \%)$ & 0.30 \\
\hline Diarrhea & $2(10 \%)$ & $11(13.3 \%)$ & 1.00 \\
\hline Onset to FMC (day) & $3(3-4)$ & $3(2-6)$ & 0.62 \\
\hline Cardiogenic shock & & & $<0.0001$ \\
\hline Presented when admission & $17(85 \%)$ & 0 & \\
\hline Developed after admission & $3(15 \%)$ & 0 & \\
\hline IABP use & $17(85 \%)$ & 0 & $<0.0001$ \\
\hline ECMO use & $6(30 \%)$ & 0 & $<0.0001$ \\
\hline Temporary pacemaker use & $11(55 \%)$ & $6(7.2 \%)$ & $<0.0001$ \\
\hline Hospitalization stay (day) & $15(10-24)$ & $9(7-12)$ & 0.003 \\
\hline Fever & $12(60 \%)$ & $42(50.6 \%)$ & 0.31 \\
\hline Temperature $\left({ }^{\circ} \mathrm{C}\right)$ & $36.5(36.1-37.2)$ & $36.5(36.3-36.9)$ & 0.73 \\
\hline $\mathrm{SBP}(\mathrm{mmHg})$ & $90.5 \pm 9.70$ & $117.6 \pm 15.8$ & $<0.0001$ \\
\hline $\mathrm{DBP}(\mathrm{mmHg})$ & $61.7 \pm 12.50$ & $71.2 \pm 9.78$ & 0.0004 \\
\hline HR (beats/min) & $95.1 \pm 23.60$ & $88.7 \pm 17.25$ & 0.17 \\
\hline $\mathrm{SI}$ & $1.0(0.82-1.81)$ & $0.74(0.63-0.91)$ & $<0.0001$ \\
\hline RR (per min) & $20(19-25)$ & 20 (19-20) & 0.37 \\
\hline WBC $(\times 109 / L)$ & $8.1(7.1-11.1)$ & $7.3(5.6-9.3)$ & 0.09 \\
\hline Neutrophil (\%) & $76.2 \pm 10.51$ & $68.8 \pm 11.88$ & 0.01 \\
\hline Lymphocyte (\%) & $15.4 \pm 9.17$ & $21.0 \pm 9.07$ & 0.02 \\
\hline BNP (pg/ml) & $686(360-1090)$ & $78(27-643)$ & 0.0001 \\
\hline Peak CKMB (U/L) & $80(40-115)$ & $32(14-52)$ & 0.0001 \\
\hline PeakTnT (ug/L) & $4.41(2.59-10)$ & $0.63(0.23-1.44)$ & $<0.0001$ \\
\hline CRP (mg/L) & $64.0(29.7-112.2)$ & $12(3.6-63.3)$ & 0.006 \\
\hline $\mathrm{ESR}(\mathrm{mm} / \mathrm{h})$ & $34(23-55)$ & $27(9-39)$ & 0.22 \\
\hline $\operatorname{ALT}(\mathrm{U} / \mathrm{L})$ & $184(56-1109)$ & $31(22-60)$ & $<0.0001$ \\
\hline $\mathrm{AST}(\mathrm{U} / \mathrm{L})$ & $302(69-1197)$ & $47(28-87)$ & $<0.0001$ \\
\hline TB (umol/L) & $12.1(6.9-16.6)$ & $12.1(8.8-15.9$ & 0.58 \\
\hline DB (umol/L) & $4.4(2.3-6.8)$ & $3.6(2.6-4.7)$ & 0.28 \\
\hline BUN (mmol/L) & $7.0(4.8-14.2)$ & $4.2(3.5-5.3)$ & $<0.0001$ \\
\hline $\mathrm{sCr}(\mathrm{umol} / \mathrm{L})$ & $73(53-152)$ & 65 (56-76) & 0.19 \\
\hline UA (umol/L) & $361(325-532)$ & $340(308-413)$ & 0.17 \\
\hline
\end{tabular}

The continuous data were presented as mean \pm standard deviation or median (interquartile range) The categorical data were presented as frequency (percentage)

$B M I$ body mass index, IABP intra-aortic balloon pump, $E C M O$ extracorporeal membrane oxygenation, $S B P$ systolic blood pressure, $D B P$ diastolic blood pressure, $H R$ heart rate, $S /$ shock index, $R R$ respiratory rate, $W B C$ white blood cell count, $B N P$ brain natriuretic peptide, CKMB creatinine kinase MB subtype, $T n T$ troponin T, $C R P$ C-reactive protein, ESR erythrocyte sedimentation rate, $A L T$ alanine transaminase, $A S T$ aspartate transaminase, $T B$ total bilirubin, $D B$ direct bilirubin, $B U N$ blood urea nitrogen, sCr serum creatinine, $U A$ uric acid 
Table 2 ECG and UCG characteristics of patient cohort

\begin{tabular}{|c|c|c|c|}
\hline & FM group $(n=20)$ & NFM group $(n=83)$ & $P$ value \\
\hline \multicolumn{4}{|l|}{ ECG parameters } \\
\hline PR interval (ms) & $189(156-210)$ & $154(136-174)$ & 0.0006 \\
\hline QRS complex duration (ms) & 104 (89-133) & $90(82-100)$ & 0.005 \\
\hline Wide QRS complex & $14(70 \%)$ & $7(8.9 \%)$ & $<0.0001$ \\
\hline QTc interval (ms) & $432(376-464)$ & $401(387-421)$ & 0.14 \\
\hline QRS-T angle (degree) & $51(35-97)$ & $34(22-75)$ & 0.08 \\
\hline Pathological Q wave & $15(75 \%)$ & $9(11.5 \%)$ & $<0.0001$ \\
\hline ST segment depression & $8(40 \%)$ & $8(9.6 \%)$ & 0.001 \\
\hline ST segment elevation & $12(60 \%)$ & $16(19.3 \%)$ & 0.003 \\
\hline T wave inverse & $16(80 \%)$ & $19(22.9 \%)$ & $<0.0001$ \\
\hline Ventricular arrhythmia & $10(50 \%)$ & $5(6.3 \%)$ & $<0.0001$ \\
\hline Sinus arrest & $1(5 \%)$ & $4(5.1 \%)$ & 1.00 \\
\hline First degree AVB & $10(50 \%)$ & $5(6.3 \%)$ & $<0.0001$ \\
\hline Second degree AVB & $1(5 \%)$ & $2(2.5 \%)$ & 0.50 \\
\hline High degree AVB & $2(10 \%)$ & $2(2.5 \%)$ & 0.18 \\
\hline Third degree AVB & $8(40 \%)$ & $6(7.6 \%)$ & 0.001 \\
\hline \multicolumn{4}{|l|}{ UCG parameters } \\
\hline IVST (cm) & $0.9(0.8-1.0)$ & $0.8(0.8-0.9)$ & 0.07 \\
\hline LVPWT (cm) & $0.9(0.8-1.0)$ & $0.8(0.8-0.9)$ & 0.07 \\
\hline $\operatorname{LAD}(\mathrm{cm})$ & $3.6 \pm 0.51$ & $3.5 \pm 0.40$ & 0.50 \\
\hline $\mathrm{AoD}(\mathrm{cm})$ & $2.6 \pm 0.27$ & $2.8 \pm 0.29$ & 0.18 \\
\hline LVEDD (cm) & $5.0 \pm 0.51$ & $5.0 \pm 0.44$ & 0.90 \\
\hline LVESD (cm) & $4.1 \pm 0.67$ & $3.6 \pm 0.59$ & 0.001 \\
\hline LVEF (\%) & $37(29-46)$ & $57(50-60)$ & $<0.0001$ \\
\hline $\operatorname{PAP}(\mathrm{mmHg})$ & $30(23-33)$ & $25(21-28)$ & 0.04 \\
\hline
\end{tabular}

The continuous data were presented as mean \pm standard deviation or median (interquartile range)

The categorical data were presented as frequency (percentage)

ECG electrocardiography, UCG ultracardiography, AVB atrioventricular block, IVST intraventricular septal thickness, LVPWT left ventricular posterior wall thickness, LAD left atrial diameter, AoD aorta diameter, LVEDD left ventricular end-distolic diameter, LVESD left ventricular end-systolic diameter, LVEF left ventricular ejection fraction, $P A P$ pulmonary artery pressure

were depicted for comparing the diagnostic capability (Fig. 4). The AUC of ECG score was significantly larger than the AUC of SI $(P<0.05)$, but similar to that of LVEF. It indicated that ECG score seemed to be a more superior diagnostic index for CS.

\section{Cutpoint of the ECG score}

The sensitivity, specificity, PPV, NPV and accuracy of the ECG score were listed in the Table 6. The identification of an appropriate cutoff value should consider the life-threatening situation of the CS, which needed a relative high NPV to reduce the false negative rate. ECG score below 500 points had quite a high NPV but PPV was a little more lower, which might increase the misdiagnosis. Thus, we set the suitable cutpoint of ECG score at 560 points with nearly $95 \%$ NPV and $74 \%$ PPV.

\section{Discussion}

Acute myocarditis involves the degenerative or necrotic changes in the myocytes due to various etiologies. FM accounts for about 30\% in the hospitalized patients of acute myocarditis [5]. Rapid progress of haemodymamical disorder and CS are the main characteristics of FM, which may lead to death in several hours or days if the patients are not treated efficiently. So far, the measurements for quick recognition of CS are limited. However, we have attempted to develop a new measurement using ECG to diagnose CS in the present of acute myocarditis.

In the acute phase, the depolarization, repolarization and electric conduction of the myocardial tissue will change to different extent. Sometimes the changes are too slight to be reflected on ECG. Many changes of ECG are nonspecific and not decisive for diagnosis. However, some morphological or electric alterations are more likely to occur in FM patients. ACS-like ECG usually includes pathological Q wave, ST segment elevation/ 
Table 3 Univariate logistic analysis for CS

\begin{tabular}{|c|c|c|c|}
\hline Variables & Odd ratio & $95 \% \mathrm{Cl}$ & $P$ value \\
\hline Age & 1.02 & {$[0.991 .05]$} & 0.13 \\
\hline $\begin{array}{l}\text { Sex } \\
\text { (Male:0, Female:1) }\end{array}$ & 2.94 & [1.08 8.04] & 0.04 \\
\hline BMl & 1.15 & {$\left[\begin{array}{lll}0.95 & 1.40\end{array}\right]$} & 0.15 \\
\hline Hypertension & 0.49 & [0.06 4.19] & 0.52 \\
\hline Smoker & 2.06 & {$[0.567 .51]$} & 0.28 \\
\hline Drinker & 0.68 & {$[0.085 .95]$} & 0.72 \\
\hline BMl & 1.15 & {$[0.951 .39]$} & 0.15 \\
\hline $\begin{array}{l}\text { SI } \\
\text { (per } 0.1 \text { increase) }\end{array}$ & 1.06 & {$[1.262 .04]$} & $<0.0001$ \\
\hline $\begin{array}{l}\text { CRP } \\
\text { (per } 1.0 \mathrm{mg} / \mathrm{L} \text { increase) }\end{array}$ & 1.01 & {$[0.991 .01]$} & 0.06 \\
\hline $\begin{array}{l}\text { PR interval } \\
\text { (per } 10 \text { ms increase) }\end{array}$ & 1.29 & {$[1.101 .51]$} & 0.002 \\
\hline $\begin{array}{l}\text { QRS complex duration } \\
\text { (per } 10 \text { ms increase) }\end{array}$ & 1.59 & {$[1.222 .06]$} & 0.001 \\
\hline $\begin{array}{l}\text { QTc interval } \\
\text { (per } 10 \mathrm{~ms} \text { increase) }\end{array}$ & 1.11 & {$\left[\begin{array}{lll}0.98 & 1.27\end{array}\right]$} & 0.11 \\
\hline $\begin{array}{l}\text { QRS-T angle } \\
\text { (per } 10 \text { degree increase) }\end{array}$ & 1.07 & {$\left[\begin{array}{lll}0.97 & 1.19\end{array}\right]$} & 0.19 \\
\hline Pathological Q wave & 23.00 & {$[6.7478 .5]$} & $<0.0001$ \\
\hline ST segment elevation & 6.28 & {$[2.2017 .9]$} & 0.001 \\
\hline ST segment depression & 6.25 & {$\left[\begin{array}{lll}1.97 & 19.8\end{array}\right]$} & 0.002 \\
\hline Twave inverse & 13.50 & {$[4.02$ 45.2] } & $<0.0001$ \\
\hline Ventricular arrhythmia & 14.80 & {$[4.20$ 52.2] } & $<0.0001$ \\
\hline Second to Third degree AVB & 3.93 & [1.60 9.68] & $<0.01$ \\
\hline $\begin{array}{l}\text { IVST } \\
\text { (per } 0.1 \mathrm{~cm} \text { increase) }\end{array}$ & 1.34 & {$[0.981 .82]$} & 0.06 \\
\hline $\begin{array}{l}\text { LVEF } \\
\text { (per 5\% increase) }\end{array}$ & 0.49 & {$[0.360 .67]$} & $<0.0001$ \\
\hline PAP & 1.09 & [0.99 1.19] & 0.06 \\
\hline
\end{tabular}

CS cardiogenic shock, CRP C-reactive protein, BMI body mass index, SI shock index, AVB atrioventricular block, IVST intraventricular septal thickness, LVEF left ventricular ejection fraction, PAP pulmonary artery pressure

Table 4 Multivariate analysis of ECG parameters for CS

\begin{tabular}{lccc}
\hline Variables & Odd ratio & $\mathbf{9 5 \% ~ C l}$ & P value \\
\hline $\begin{array}{l}\text { PR + QRS interval } \\
\text { (per 10 ms increase) }\end{array}$ & 1.29 & {$[1.071 .55]$} & 0.008 \\
Pathological Q wave & 30.3 & {$[4.40208 .1]$} & 0.003 \\
Ventricular arrhythmia & 26.8 & {$[2.99238 .6]$} & 0.001 \\
\hline
\end{tabular}

depression and $\mathrm{T}$ wave inverse. The incidence of pathological Q wave varies in different studies $[12,13,19]$. In the current study, the incidence of pathological Q wave is $75 \%$ in FM group, which is higher than the previous studies. It is usually associated with the myocardial transmural lesion and correspond with the distribution of late gadolinium enhancement (LGE) on CMR [20,

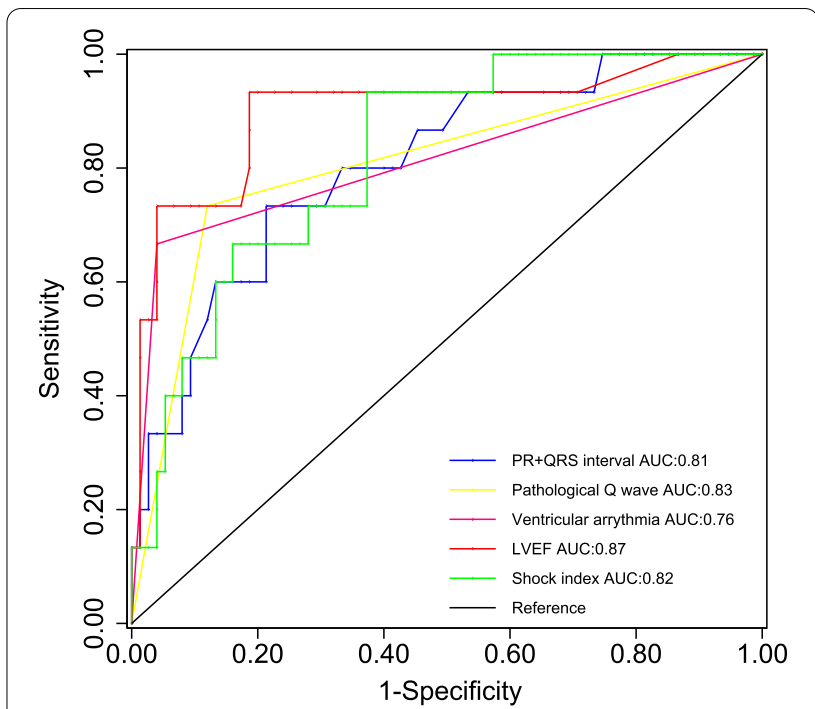

Fig. 2 Comparison of the diagnostic capability for the different indices. There was no significant difference among the area under the five ROC curves

21]. Furthermore, the presence of pathological Q wave is identified as a predictor of poor prognosis in FM patients [22]. ST-T alteration is present both in FM and NFM patients. We have reported a much higher incidence of ST segment elevation/depression and T wave inverse in FM group. Different from Q wave, ST segment elevation is less helpful in evaluating the location of myocardial tissue but can be used as a quick assessment of the extent of myocardial injury [23]. As to ST segment depression, it has been suggested an early sign of FM [15]. However, ST segment elevation/depression and $\mathrm{T}$ wave inverse were not identified relevant to the CS in the current study.

PR interval prolongation and wide QRS complex are suggestive of impairment of conduction system and the prevalence is usually in proportion to the severity of the conditions. We found that the PR interval and QRS complex duration in FM patients were both significantly prolonged than that in NFM patients. The incidence of wide QRS complex in our study was as high as $70 \%$, which was very close to the previous findings [14]. And we also found these two index was useful in quick diagnosis of CS. Ventricular arrhythmia and AVB are both reported common in FM $[14,24]$, which has been supported by our findings. Nonetheless, tachyarrhythmia is more common than bradyarrhythmia [17]. Ventricular arrhythmia is considered related to myocardial edema, scar and severe impairment of cardiac function. LEG on CMR can increase the likelihood of ventricular arrhythmia $[25,26]$. The current study found the ventricular arrhythmia were more likely to occur in FM patients but also occurred in a small part of NFM patients, which is consistent with 


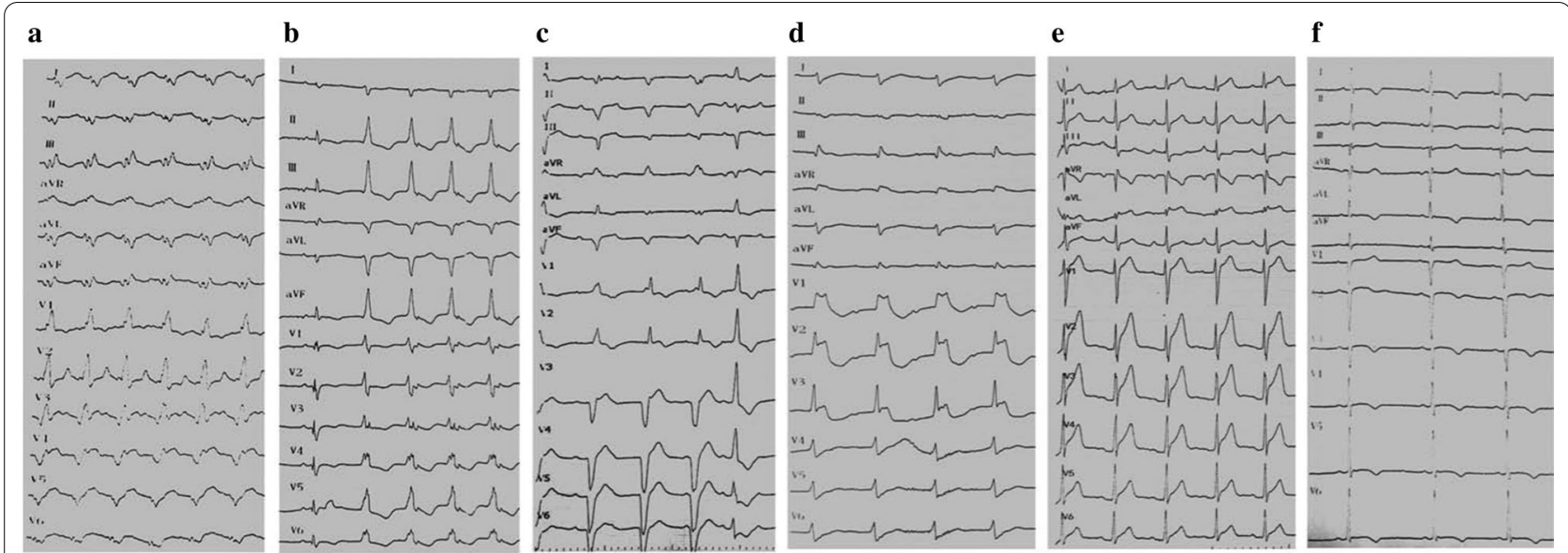

Fig. 3 a FM patient. PR interval 150 ms, QRS complex 140 ms, with pathological Q wave, without ventricluar arrhythmia. ECG score $=580$ points. b FM patient. PR interval $160 \mathrm{~ms}$, QRS complex $130 \mathrm{~ms}$, with ventricular tachycardia, without pathological Q wave. ECG score $=580$ points. c FM patient. PR interval $170 \mathrm{~ms}$, QRS complex $110 \mathrm{~ms}$, with accelerated idoventricular rhythm, without pathological Q wave, ECG score = 560 points. d FM patient. PR interval $280 \mathrm{~ms}$, QRS complex $240 \mathrm{~ms}$, without ventricular arrhythmia and pathological Q wave. ECG score $=520$ points. e NFM patient. PR interval $180 \mathrm{~ms}$, QRS complex $100 \mathrm{~ms}$, without ventricular arrhythmia and pathological Q wave. ECG score $=280$ points. f NFM patient. PR interval 140 ms, QRS complex 90 ms, without ventricular arrhythmia and pathological Q wave. ECG score $=230$ points

Table 5 Multivariate regression model fit for the derived variable

\begin{tabular}{|c|c|c|c|c|}
\hline Model & Variables & Odd ratio & $95 \% \mathrm{Cl}$ & $P$ value \\
\hline \multirow{2}{*}{$\begin{array}{l}\text { Model } 1 \\
\text { Age + Sex + SI } \\
+ \text { LVEF + IVST } \\
+ \text { PAP + ECG } \\
\text { score }\end{array}$} & $\begin{array}{l}\text { ECG Score } \\
\text { (per } 10 \text { increase) }\end{array}$ & 1.13 & [1.07 1.09] & 0.002 \\
\hline & $\begin{array}{l}\text { LVEF } \\
\text { (per 5\% increase) }\end{array}$ & 0.53 & {$\left[\begin{array}{lll}0.33 & 0.85\end{array}\right]$} & 0.009 \\
\hline \multirow{2}{*}{$\begin{array}{l}\text { Model } 2 \\
\text { Age + Sex + SI } \\
+ \text { IVST + PAP } \\
+ \text { ECG score }\end{array}$} & $\begin{array}{l}\text { ECG Score } \\
\text { (per } 10 \text { increase) }\end{array}$ & 1.11 & {$\left[\begin{array}{ll}1.06 & 1.18\end{array}\right]$} & $<0.0001$ \\
\hline & $\begin{array}{l}\text { SI } \\
\text { (per } 0.1 \text { increase) }\end{array}$ & 1.42 & [1.05 2.04] & 0.049 \\
\hline
\end{tabular}

SI shock index, IVST intraventricular septal thickness, LVEF left ventricular ejection fraction, PAP pulmonary artery pressure

the former study [24]. Ventricular arrhythmia, wide QRS complex and AVB are sometimes present in a single patient alternatively, even before the emergence of haemodynamical disorder. Seen in this light, certain morphological changes on ECG may be helpful to early and rapid recognition of CS.

The ECG score was generated completely original. PR interval and QRS complex duration are the main body of the score. Occurrence of ventricular arrhythmia and pathological $Q$ wave will provide weight for the main body. It integrates different relevant factors of the ECG into a single index and can be used conveniently.

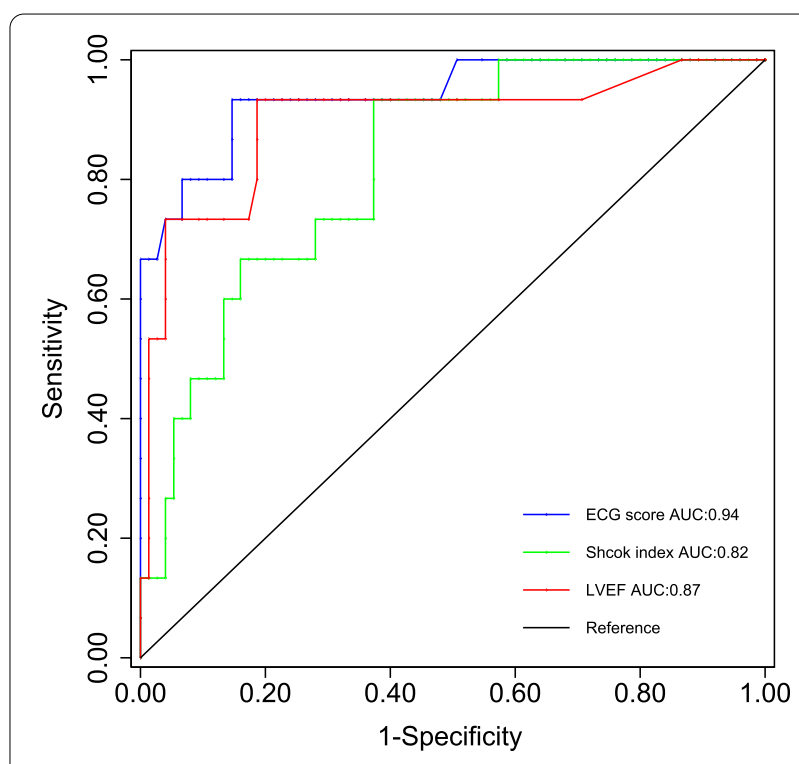

Fig. 4 Assessment of the diagnostic capability of ECG score. The area under the ROC curve of ECG score was similar to that of LVEF, but significantly larger than that of shock index $(P<0.05)$

ECG score is independent of LVEF and SI in diagnosing CS. It also presented a superior diagnostic capability in comparison with LVEF and SI. From Table 6, we found ECG score had a high NPV and therefore suitable for exclusion diagnosis. When ECG score was beyond 560 points, the PPV was increased significantly with a slight decrease of NPV. Of note, ECG score can be calculated only when sinus rhythm is seen on ECG for measurment 
Table 6 Diagnostic capability of ECG score for CS at different cutpoints

\begin{tabular}{|c|c|c|c|c|c|}
\hline Cutpoint & Sensitivity (\%) & Specificity (\%) & Accuracy (\%) & PPV (\%) & NPV (\%) \\
\hline 400 & 94.4 & 82.9 & 85.1 & 58.6 & 98.4 \\
\hline 440 & 94.4 & 85.5 & 87.2 & 59.3 & 95.6 \\
\hline 480 & 83.3 & 86.8 & 86.2 & 65.2 & 95.8 \\
\hline 520 & 83.3 & 89.5 & 88.3 & 65.2 & 95.8 \\
\hline 560 & 77.8 & 93.4 & 90.4 & 73.7 & 94.7 \\
\hline 600 & 66.7 & 96.1 & 90.4 & 84.6 & 91.4 \\
\hline 640 & 61.1 & 97.4 & 90.4 & 91.7 & 91.5 \\
\hline 680 & 55.6 & 100.0 & 91.5 & 100.0 & 90.1 \\
\hline
\end{tabular}

CS cardiogenic shock, $P P V$ positive predictive value, NPV negative predictive value

of PR interval. If the ECG presents ventricular arrhythmia (such as ventricular tachycardia or AIVR) without sinus rhythm, it provides more powerful evidence for the diagnosis of fulminant myocarditis and ECG score is not necessary under this circumstance. Besides, subgroup analysis, such age subgroup, sex subgroup, was not performed due to relative small sample size. Thus, it was unclear whether the ECG score as a new diagnostic index was age specific or sex specific. We did even not know whether this score was applicable to the children because the study cohort did not contain children patients. However, these issues are quite important and meaningful. Particularly, it is worthy of further investigation whether the cutpoint varies in different age range or different sex.

In summary, the ECG score, derived from the parameters of ECG, have possessed an outstanding diagnostic capability of $C S$ caused by acute myocarditis. It may be a reliable substitution for LVEF or SI and can be used for a early recognition of the haemodynamical derangement.

\section{Limitations}

The current study is a single center and retrospective analysis. There are several limitations. First, the sample size is a little bit small. When we performed diagnostic statistics, there are breakpoints between the two adjacent cutoff values. We cannot learn the diagnostic indices at these breakpoints. Second, subgroup analysis could not be carried out due to the small sample size. Whether the ECG score also works in different age group or sex group is unknown. Whether the cutpoint varies in different subgoups is also unclear. Third, the serum lactate level is also an useful biomarker for early assessment of peripheral perfusion. Nonetheless, in our study, most of the haemodynamical stable patients did not have the lactate test. The comparison of ECG score with lactate could not be carried out. Fourth, endomyocardial biopsy was not available in our center. Despite all the patients were caused by infection, we could not evaluate the relationship between pathological type and severity of the conditions. The last but not least, the diagnostic capability of ECG score is acquired from the single center, retrospective study. We need multicenter, prospective trials with larger sample size to verify the conclusions.

\begin{abstract}
Abbreviations
ACEl: Angiotensin-converting enzyme inhibitor; ACS: Acute coronary syndrome; AIVR: Accelerated idioventricular rhythm; ARB: Angiotensin receptor blockade; AUC: Area under curve; AVB: Atrioventricular block; BNP: Brain natriuretic peptide; CCU: Cardiac care unit; CHD: Coronary heart disease; CMR: Cardiac magnetic resonance; CS: Cardiogenic shock; ECG: Electrocardiography; ECMO: Extracorporeal membrane oxygenation; ED: Emergency department; FM: Fulminant myocarditis; FMC: First medical contact; IABP: Intra-aortic balIoon pump; IQR: Interquartile range; IVST: Intraventricular septal thickness; LGE: Late gadolinium enhancement; LVEDD: Left ventricular end-diastolic diameter; LVEF: Left ventricular ejection fraction; LVESD: Left ventricular end-systolic diameter; LVPWT: Left ventricular posterior wall thickness; NFM: Non fulminant myocarditis; NPV: Negative predictive value; PPV: Positive predictive value; ROC: Receiver-operating characteristics; SI: Shock index; UCG: Ultracardiography; VT: Ventricular tachycardia.
\end{abstract}

\section{Acknowledgements \\ Not applicable.}

\section{Authors' contributions}

DY: data collection and manuscript writing. YD: manuscript writing and data analysis. HW: data collection and management. JZC: patients follow-up and data analysis. JMZ: manuscript revision and data review. ZHW data analysis, data review and manuscript review. All authors have read and approved the manuscript.

\section{Funding}

This study was supported by the following funding: The National Natural Science Foundation of China (No. 81700392), hosted by Zhonghai Wei. The National Natural Science Foundation of China (No. 81700389), hosted by Jianzhou Chen. The Municipal Medical Science Technology Development Foundation of Nanjing (No. YKK19063), hosted by Zhonghai Wei. The Municipal Medical Science Technology Development Foundation of Nanjing (No. YKK17085), hosted by Zhonghai Wei. The funding are used to pay for the service provided by the investigators, including data collection, data analysis and writing the manuscript. The funding are also used to pay the publication charges of the manuscript.

\section{Availability of data and materials}

The information and data of the study population were acquired from Hospital Information System and were recorded manually in EXCEL to form the database. The datasets analyzed during the current study are not publicly available due to the protection of the individual privacy but are available from the corresponding author on reasonable request. 


\section{Ethics approval and consent to participate}

The study has been approved by the Medical Ethics Committee of Nanjing Drum Tower Hospital, Medical School of Nanjing University (2019-190-01). All the participants were consent to participate with the verbal consent and it has been approved by the ethics committee. The database is not open to public, thus the individual privacy of the participants could be well protected.

\section{Consent for publication}

Not applicable.

\section{Competing interests}

The authors declare that they have no competing interests.

\section{Author details}

${ }^{1}$ Department of Cardiology, Drum Tower Hospital, Medical School of Nanjing University, Nanjing 210008, China. ${ }^{2}$ Department of Cardiology, Yizheng Hospital, Nanjing Drum Tower Hospital Group, Yizheng 211900, China.

Received: 14 August 2020 Accepted: 24 November 2020

Published online: 01 December 2020

\section{References}

1. Kyto V, Saraste A, Voipio-Pulkki LM, Saukko P. Incidence of fatal myocarditis: a population-based study in Finland. Am J Epidemiol. 2007;165:570-4.

2. Global Burden of Disease Study 2013 Collaborators. Global, regional, and national incidence, prevalence, and years lived with disability for 301 acute and chronic diseases and injuries in 188 countries, 1990-2013: a systematic analysis for the global burden of disease study 2013. Lancet. 2015:386:743-800.

3. Sharma AN, Stultz JR, Bellamkonda N, Amsterdam EA. Fulminant myocarditis: epidemiology, pathogenesis, diagnosis, and management. Am J Cardiol. 2019;124:1954-60.

4. McCarthy RE 3rd, Boehmer JP, Hruban RH, Hutchins GM, Kasper EK, Hare JM, Baughman KL. Long-term outcome of fulminant myocarditis as compared with acute (nonfulminant) myocarditis. N Engl J Med. 2000;342:690-5.

5. Ammirati E, Cipriani M, Lilliu M, Sormani P, Varrenti M, Raineri C, Petrella D, Garascia A, Pedrotti P, Roghi A, Bonacina E, Moreo A, Bottiroli M, Gagliardone MP, Mondino M, Ghio S, Totaro R, Turazza FM, Russo CF, Oliva F, Camici PG, Frigerio M. Survival and left ventricular function changes in fulminant versus nonfulminant acute myocarditis. Circulation. 2017:136:529-45.

6. Ishida K, Wada H, Sakakura K, Kubo N, Ikeda N, Sugawara Y, Ako J, Momomura S. Long-term follow-up on cardiac function following fulminant myocarditis requiring percutaneous extracorporeal cardiopulmonary support. Heart Vessels. 2013;28:86-90.

7. Bircan A, Karadeniz N, Ozden A, Cakir M, Varol E, Oyar O, Ozaydin M. A simple clinical model composed of ECG, shock index, and arterial blood gas analysis for predicting severe pulmonary embolism. Clin Appl Thromb Hemost. 2011;17:188-96.

8. Rappaport LD, Deakyne S, Carcillo JA, McFann K, Sills MR. Age- and sexspecific normal values for shock index in national health and nutrition examination survey 1999-2008 for ages 8 years and older. Am J Emerg Med. 2013;31:838-42.

9. Keller AS, Kirkland LL, Rajasekaran SY, Cha S, Rady MY, Huddleston JM Unplanned transfers to the intensive care unit: the role of the shock index. J Hosp Med. 2010;5:460-5.

10. Sankaran P, Kamath AV, Tariq SM, Ruffell H, Smith AC, Prentice P, Subramanian DN, Musonda P, Myint PK. Are shock index and adjusted shock index useful in predicting mortality and length of stay in community-acquired pneumonia? Eur J Intern Med. 2011;22:282-5.

11. Buttà C, Zappia L, Laterra G, Roberto M. Diagnostic and prognostic role of electrocardiogram in acute myocarditis: A comprehensive review. Ann Noninvasive Electrocardiol. 2020:25:12726.

12. Demangone D. ECG manifestations: noncoronary heart disease. Emerg Med Clin N Am. 2006:24:113-31.
13. Punja M, Mark DG, McCoy JV, Javan R, Pines JM, Brady W. Electrocardiographic manifestations of cardiac infectious-inflammatory disorders. Am J Emerg Med. 2010;28:364-77.

14. Sawamura A, Okumura T, Ito M, Ozaki Y, Ohte N, Amano T, Murohara T. Prognostic value of electrocardiography in patients with fulminant myocarditis supported by percutaneous venoarterial extracorporeal membrane oxygenation - analysis from the change pump study. Circ J. 2018;82:2089-95

15. Wang Z, Wang Y, Lin H, Wang S, Cai X, Gao D. Early characteristics of fulminant myocarditis vs non-fulminant myocarditis: a meta-analysis. Medicine (Baltimore). 2019;98:e14697.

16. Caforio AL, Pankuweit S, Arbustini E, Basso C, Gimeno-Blanes J, Felix SB, Fu M, Helio T, Heymans S, Jahns R, Klingel K, Linhart A, Maisch B, McKenna W, Mogensen J, Pinto YM, Ristic A, Schultheiss HP, Seggewiss H, Tavazzi L, Thiene G, Yilmaz A, Charron P, Elliott PM. Current state of knowledge on aetiology, diagnosis, management, and therapy of myocarditis: a position statement of the European Society of Cardiology Working Group on myocardial and pericardial diseases. Eur Heart J. 2013;34(2636-2648):2648a-2 $648 d$.

17. Kociol RD, Cooper LT, Fang JC, Moslehi JJ, Pang PS, Sabe MA, Shah RV, Sims DB, Thiene G, Vardeny O. Recognition and initial management of fulminant myocarditis: a scientific statement from the American Heart Association. Circulation. 2020;141:e69-92.

18. MCMurray JJ, Adamopoulos S, Anker SD, Auricchio A, Bohm M, Dickstein K, Falk V, Filippatos G, Fonseca C, Gomez-Sanchez MA, Jaarsma T, Kober L, Lip GY, Maggioni AP, Parkhomenko A, Pieske BM, Popescu BA, Ronnevik PK, Rutten FH, Schwitter J, Seferovic P, Stepinska J, Trindade PT, Voors AA, Zannad F, Zeiher A. ESC guidelines for the diagnosis and treatment of acute and chronic heart failure 2012: the task force for the diagnosis and treatment of acute and chronic heart failure 2012 of the European Society of Cardiology. Developed in collaboration with the heart failure association (HFA) of the ESC. Eur Heart J. 2012;33:1787-847.

19. Nakashima $H$, Honda $Y$, Katayama T. Serial electrocardiographic findings in acute myocarditis. Intern Med. 1994;33:659-66.

20. Jhamnani S, Fuisz A, Lindsay J. The spectrum of electrocardiographic manifestations of acute myocarditis: an expanded understanding. J Electrocardiol. 2014;47:941-7.

21. Deluigi CC, Ong P, Hill S, Wagner A, Kispert E, Klingel K, Kandolf R, Sechtem U, Mahrholdt H. ECG findings in comparison to cardiovascular MR imaging in viral myocarditis. Int J Cardiol. 2013;165:100-6.

22. Ginsberg F, Parrillo JE. Fulminant myocarditis. Crit Care Clin. 2013;29:465-83.

23. Nucifora G, Miani D, Di Chiara A, Piccoli G, Artico J, Puppato M, Slavich G, De Biasio M, Gasparini D, Proclemer A. Infarct-like acute myocarditis: relation between electrocardiographic findings and myocardial damage as assessed by cardiac magnetic resonance imaging. Clin Cardiol. 2013:36:146-52.

24. Graner M, Lommi J, Kupari M, Raisanen-Sokolowski A, Toivonen L. Multiple forms of sustained monomorphic ventricular tachycardia as common presentation in giant-cell myocarditis. Heart. 2007;93:119-21.

25. Ammirati E, Cipriani M, Moro C, Raineri C, Pini D, Sormani P, Mantovani R, Varrenti M, Pedrotti P, Conca C, Mafrici A, Grosu A, Briguglia D, Guglielmetto S, Perego GB, Colombo S, Caico SI, Giannattasio C, Maestroni A, Carubelli V, Metra M, Lombardi C, Campodonico J, Agostoni P, Peretto G, Scelsi L, Turco A, Di Tano G, Campana C, Belloni A, Morandi F, Mortara A, Ciro A, Senni M, Gavazzi A, Frigerio M, Oliva F, Camici PG. Clinical presentation and outcome in a contemporary cohort of patients with acute myocarditis. Circulation. 2018;138:1088-99.

26. Sanguineti F, Garot P, Mana M, O'H-Ici D, Hovasse T, Unterseeh T, Louvard Y, Troussier X, Morice MC, Garot J. Cardiovascular magnetic resonance predictors of clinical outcome in patients with suspected acute myocarditis. J Cardiovasc Magn Reson. 2015;17:78.

\section{Publisher's Note}

Springer Nature remains neutral with regard to jurisdictional claims in published maps and institutional affiliations. 